\title{
Anti-angiogenic effect of hexahydrocurcumin in rat corneal neovascularization
}

\section{Chien-Hsing Lee}

\author{
Kaohsiung Medical University, Taiwan
}

Aim This study was to investigate the anti-angiogenic effect of hexahydrocurcumin (HHC) to evaluate gene (p-basic fibroblast growth factor (bFGF)-SAINT-18 \& p-vascular endothelial growth factor (VEGF)-SAINT-18 complex)-induced corneal neovascularization (CorNV) in rats.

Methods CorNV was induced in 24 eyes of 24 rats. Four groups (Group A: 0 lg, B: $0.01 \mathrm{lg}$, C: $0.1 \mathrm{lg}$, and D: $1 \mathrm{lg}$ ) of HHC were prepared and implanted into the rat subconjunctival substantia propria $1.5 \mathrm{~mm}$ from the limbus at temporal side. The $1 \mathrm{lg}$ of p-bFGFSAINT-18 \& p-VEGF-SAINT-18 complex were prepared and implanted into the rat corneal stroma $1.5 \mathrm{~mm}$ from the limbus at the same side. Inhibition of CorNV was observed and quantified from day 1 to day 60. bFGF and VEGF protein expression were analyzed by biomicroscopic examination, western blot analysis, and immunohistochemistry.

Results Subconjunctival injection by 1 lg HHC successfully inhibited gene-induced CorNV in rats. bFGF and VEGF protein expression were reduced after 6 days. Meanwhile, the reduction of HLA-DR expression was detected.

Conclusions Our study showed that the HHC might provide an important anti-angiogenesis factor to inhibit CorNV development at the corneal experimental

angiogenesis model. 